\title{
Incoherent radar spectra in the auroral ionosphere in the presence of a large electric field: The effect of $\mathrm{O}^{+}-\mathrm{O}^{+}$Coulomb collisions
}

\author{
I. A. Barghouthi \\ Department of Physics, AL-Quds University, P.O. Box 20002, Jerusalem, Palestine
}

(Received June 13, 2004; Revised May 7, 2005; Accepted May 9, 2005)

\begin{abstract}
We have used Monte Carlo simulations of $\mathrm{O}^{+}$velocity distributions in the high latitude $\mathrm{F}$ - region to improve the calculation of incoherent radar spectra in auroral ionosphere. The Monte Carlo simulation includes ionneutral, $\mathrm{O}^{+}-\mathrm{O}$ collisions (resonant charge exchange and polarization interaction) as well as $\mathrm{O}^{+}-\mathrm{O}^{+}$Coulomb self-collisions. At high altitudes, atomic oxygen $\mathrm{O}$ and atomic oxygen ion $\mathrm{O}^{+}$dominate the composition of the auroral ionosphere and consequently, the influence of $\mathrm{O}^{+}-\mathrm{O}^{+}$Coulomb collisions becomes significant. In this study we consider the effect of $\mathrm{O}^{+}-\mathrm{O}^{+}$Coulomb collisions on the incoherent radar spectra in the presence of large electric field $\left(100 \mathrm{mVm}^{-1}\right)$. As altitude increases (i.e. the ion-to-neutral density ratio increases) the role of $\mathrm{O}^{+}-\mathrm{O}^{+}$Coulomb self-collisions becomes significant, therefore, the one-dimensional, 1-D, $\mathrm{O}^{+}$ion velocity distribution function becomes more Maxwellian and the features of the radar spectrum corresponding to nonMaxwellian ion velocity distribution (e.g. baby bottle and triple hump shapes) evolve to Maxwellian ion velocity distribution (single and double hump shapes). Therefore, $\mathrm{O}^{+}-\mathrm{O}^{+}$Coulomb self-collisions act to isotropize the 1-D $\mathrm{O}^{+}$velocity distribution by transferring thermal energy from the perpendicular direction to the parallel direction, however the convection electric field acts to drive the $\mathrm{O}^{+}$ions away from equilibrium and consequently, non-Maxwellian $\mathrm{O}^{+}$ion velocity distributions appeared. Therefore, neglecting $\mathrm{O}^{+}-\mathrm{O}^{+}$Coulomb self-collisions overestimates the effect of convection electric field.
\end{abstract}

Key words: Incoherent radar spectra, auroral ionosphere, Coulomb collision, convection electric field, Monte Carlo simulation.

\section{Introduction}

Above $200 \mathrm{~km}$, atomic oxygen $\mathrm{O}$ dominates the composition of the auroral ionosphere, and as a result of the Sun's ionizing radiation, $\mathrm{O}^{+}$ions dominate the composition of the auroral ionosphere (Salah, 1993). Several theoretical, experimental and simulation papers have investigated the $\mathrm{O}^{+}$ ion velocity distributions in the high-latitude F-region, in order to properly interpret F-region satellite and radar data (Gaimard et al., 1996). The presence of strong convection electric fields in the high latitudes F-region produces $\mathbf{E} \times \mathbf{B ~ O}^{+}$drift that can exceed the neutral O thermal velocity. Due to the interplay between $\mathbf{E} \times \mathbf{B} \mathrm{O}^{+}$drifts and $\mathrm{O}^{+}-\mathrm{O}$ interactions, the $\mathrm{O}^{+}$velocity distribution function shows significant non-Maxwellian features such as toroidal characteristics and temperature anisotropy (Barakat et al., 1983). In the case of non-Maxwellian features of the $\mathrm{O}^{+}$ velocity distribution, the incoherent radar spectra may even have a triple-humped shape instead of a double-humped one (Raman et al., 1981). Therefore, if the effects of nonMaxwellian ion velocity distributions are not taken into account when fitting the model spectra to measured ones, the obtained parameters of the auroral ionosphere will contain systematic errors. In a series of papers (Cole, 1971; St-Maurice and Schunk, 1973, 1976, 1979; Hubert, 1983,

Copy right(c) The Society of Geomagnetism and Earth, Planetary and Space Sciences (SGEPSS); The Seismological Society of Japan; The Volcanological Society of Japan; The Geodetic Society of Japan; The Japanese Society for Planetary Sciences; TERRAPUB.
1984; Barakat et al., 1983; Barakat and Hubert, 1990; Hubert and Barakat, 1990; Winkler et al., 1992; Gaimard et al., 1998), the problem that has been deeply investigated is the behavior of $\mathrm{O}^{+}$ions that are $\mathbf{E} \times \mathbf{B}$ drifting through a background of neutral particles. In these studies, the ions were assumed to collide only with neutral particles (polarization scattering and resonant charge exchange), and the effect of ion-ion Coulomb collisions was neglected. However, the ion-to-neutral density ratio increases rapidly with altitude, therefore, at high altitudes; ion-ion Coulomb collisions play a potentially important role in understanding the F-region (McCrea et al., 1993) and in deciding the shape of the ion velocity distributions.

Tereshchenko et al. (1991) and Kinzelin and Hubert (1992) studied analytically the effect of $\mathrm{O}^{+}-\mathrm{O}^{+}$Coulomb collisions on the $\mathrm{O}^{+}$ion velocity distribution function. Barghouthi et al. (1991, 1994, 2003) used a Monte Carlo simulation to find $\mathrm{O}^{+}$velocity distribution function for more realistic collision models (polarization scattering, resonant charge exchange and Coulomb collisions). This model accurately computes the moments of the $\mathrm{O}^{+}$velocity distribution and provides additional details of the distribution function that are very important in studying the incoherent radar spectra (Barakat et al., 1990).

The European incoherent scattering radar (EISCAT), with its tristatic mode of operation, was able to measure the $\mathrm{O}^{+}$temperature anisotropy. Perraut et al. (1984) used the EISCAT data to deduce that the $\mathrm{O}^{+}$ion velocity dis- 
tribution in the auroral F-region was anisotropic, that is $T_{\perp}\left(\mathrm{O}^{+}\right) / T_{\|}\left(\mathrm{O}^{+}\right)$as high as 1.5 . The EISCAT data were also used to investigate the non-Maxwellian features of the $\mathrm{O}^{+}$ ion velocity distribution (Winser et al., 1986; Lockwood et al., 1987). Winser et al. (1987) were the first to observe the distorted incoherent scatter spectra in the presence of large electric fields in the auroral F-region. Raman et al. (1981) studied the effects that non-Maxwellian ion velocity distributions produce on the ionic part of the spectrum of radar waves incoherently scattered from the disturbed highlatitude ionosphere in the upper $\mathrm{E}$ and lower F-regions. Kikuchi et al. (1989) used Monte Carlo simulations of $\mathrm{O}^{+}$ ion velocity distributions in the high-latitude F-region obtained by Barakat et al. (1983), to improve the calculation of incoherent radar spectra in the auroral ionosphere. Hubert et al. (1993) proposed a scenario for the measurement of the non-equilibrium plasma parameters from incoherent radar spectra; they deduced that at a certain angle to the geomagnetic field, the 1-D line-of-sight ion velocity distribution should be Maxwellian. Hubert and Leblanc (1997), presented new features of $\mathrm{O}^{+}$ion velocity distribution function in a background of neutral atomic oxygen; they derived the $1-\mathrm{D} \mathrm{O}^{+}$velocity distribution from the polynomial expansion whose velocity moments have been provided by Monte Carlo simulations (Barakat et al., 1983).

As mentioned earlier, analytical (Tereshchenko et al., 1991; Kinzelin and Hubert, 1992) and macroscopic (Monte Carlo) (Barghouthi et al., 1994, 2003) studies have introduced the effect of $\mathrm{O}^{+}-\mathrm{O}^{+}$Coulomb collisions on the $\mathrm{O}^{+}$ ion velocity distribution function in the auroral ionosphere. In this paper, we use the Monte Carlo simulations obtained by Barghouthi et al. (2003) to calculate the effect of $\mathrm{O}^{+}$$\mathrm{O}^{+}$Coulomb self-collisions on the corresponding incoherent scatter radar spectrum. This is the first paper to directly address the effect of $\mathrm{O}^{+}-\mathrm{O}^{+}$Coulomb collisions on the incoherent scatter radar spectrum in the presence of a large electric field on the basis of Monte Carlo simulations. Such simulations offer an improvement on analytical methods previously used in studies such as that of Tereshchenko et al. (1991), whose collision models were over-simplified compared to the approach presented here. The simulation of Coulomb collision effects on the incoherent scatter spectrum is a logical step to comparison between model predictions and experimental results, especially since many previous studies have completely neglected the influence of $\mathrm{O}^{+}$$\mathrm{O}^{+}$Coulomb collisions, leading to large systematic errors in the ion thermal velocity distribution and ion temperature, especially at F-region altitudes.

This paper is organized as follows: Section 2 presents briefly the collision models used in this study and explains how a Monte Carlo method has been used to generate the incoherent radar spectra. In Section 3 we present the basic formulation of incoherent radar spectra. We then present in Section 4 the one-dimensional $\mathrm{O}^{+}$ion velocity distribution function and incoherent radar spectra for different typical values of ion-to-neutral density ratios (i.e. different altitudes) in the presence of large convection electric field. The conclusions are presented in Section 5.

\section{Monte Carlo Model}

In this section we introduce the calculation of the $\mathrm{O}^{+}$ ion velocity distribution using a Monte Carlo simulation. Barakat et al. (1983) studied the effect of $\mathrm{O}^{+}-\mathrm{O}$ collisions (Polarization interaction and resonant charge exchange) on the $\mathrm{O}^{+}$ion velocity distributions in the presence of large electric field in the high latitude F-region. Barghouthi et al. (1994) extended the above work and included the influence of $\mathrm{O}^{+}-\mathrm{O}^{+}$Coulomb self-collisions on the $\mathrm{O}^{+}$ion velocity distribution and its moments. Recently, Barghouthi et al. (2003) investigated the behavior of $\mathrm{O}^{+}$ions in the auroral ionosphere, they obtained altitude profiles for $\mathrm{O}^{+}$ion velocity distributions, $\mathrm{O}^{+}$parallel and perpendicular temperatures, $\mathrm{O}^{+}-\mathrm{O}^{+}$and $\mathrm{O}^{+}-\mathrm{O}$ collision frequencies, and $\mathrm{O}^{+}$parallel and perpendicular temperature partition coefficients. Basically, they considered 1000 test $\mathrm{O}^{+}$ions; each $\mathrm{O}^{+}$test ion is followed for a short period of time $\Delta t$ ( $1 \%$ of the mean free time between $\mathrm{O}^{+}-\mathrm{O}^{+}$Coulomb self-collisions). Between collisions, $\mathrm{O}^{+}$ion is subjected to the Lorentz force (i.e. it moves in a circle centered at $\mathbf{E} \times \mathbf{B}$ drift). At the moment of collision (i.e. after $\Delta t$ ), the change in $\mathrm{O}^{+}$ion momentum and energy is determined and after each $\mathrm{O}^{+}-\mathrm{O}^{+}$ Coulomb self-collision the effect on $\mathrm{O}^{+}$ion velocity is calculated. After several $\mathrm{O}^{+}-\mathrm{O}^{+}$collisions a $\mathrm{O}^{+}-\mathrm{O}$ collision (Polarization interactions or resonant charge exchange) occurs. These steps are repeated for a long time (1000 mean free times) and the test $\mathrm{O}^{+}$ions are continuously monitored. Consequently, the $\mathrm{O}^{+}$velocity distribution and temperature can be calculated. We used the $\mathrm{O}^{+}$velocities to record the time spent at a given velocity bin in a two-dimensional grid with velocity coordinates parallel and perpendicular to the geomagnetic field and centered at $\mathbf{E} \times \mathbf{B}$ drift. The time spent by the $\mathrm{O}^{+}$ion in each bin of the velocity grid, divided by that bin's volume, was proportional to the $\mathrm{O}^{+}$velocity distribution at the center of that bin. Also, the data needed to compute parallel and perpendicular temperatures are accumulated. In the simulation region (i.e. F-region), the $\mathrm{O}^{+}$ collision frequency is much smaller than $\mathrm{O}^{+}$cyclotron frequency; this implies that the $\mathrm{O}^{+}$velocity distribution function is azimuthally symmetric in velocity space.

The main idea of this paper is to study the influence of $\mathrm{O}^{+}-\mathrm{O}^{+}$Coulomb collisions on the $\mathrm{O}^{+}$velocity distributions and consequently, on the incoherent radar spectra. $\mathrm{O}^{+}$$\mathrm{O}^{+}$Coulomb self-collisions are included as a binary elastic Coulomb collision, as suggested by Takisuka and Abe (1977). In particular we randomly pair the test $\mathrm{O}^{+}$ions (1000 ions) and perform a binary collision on each pair. As a result of $\mathrm{O}^{+}-\mathrm{O}^{+}$collision the magnitude of the relative speed $u=\left|\mathbf{v}_{\mathrm{O}_{1}^{+}}-\mathbf{v}_{\mathrm{O}_{2}^{+}}\right|$does not change, but its direction is altered. In a time period $\Delta t$, an $\mathrm{O}^{+}$ion makes many small angle scatterings. The accumulation of these small angle scatterings is used to compute the deflection in the relative velocity $\mathbf{u}$. The variable $\delta$ is equal to $\tan (\theta / 2)$ (where $\theta$ is the deflection angle) is chosen randomly from a Gaussian distribution with zero mean and variance $\left\langle\delta^{2}\right\rangle$ given by

$$
\left\langle\delta^{2}\right\rangle=\frac{2 \pi e^{4} n_{\mathrm{O}^{+}} \lambda}{m_{\mathrm{O}^{+}}^{2} u^{3}} \Delta t
$$

where $n_{\mathrm{O}^{+}}$is the $\mathrm{O}^{+}$ion density, $m_{\mathrm{O}^{+}}$is $\mathrm{O}^{+}$mass, $e$ is the electron charge and $\lambda$ is the Coulomb logarithm. The 
change in the $\mathrm{O}^{+}$ion velocity due to Coulomb collisions is given by

$$
\begin{aligned}
& \mathbf{v}_{\mathrm{O}_{1}^{+}}^{\prime}=\mathbf{v}_{\mathrm{O}_{1}^{+}}+\frac{\ddot{\mathbf{A}} \mathbf{u}}{2} \\
& \mathbf{v}_{\mathrm{O}_{2}^{+}}^{\prime}=\mathbf{v}_{\mathrm{O}_{2}^{+}}-\frac{\ddot{\mathrm{A}} \mathbf{u}}{2}
\end{aligned}
$$

where the primes denote velocities after Coulomb collisions. This $\mathrm{O}^{+}-\mathrm{O}^{+}$Coulomb self-collision process has been described in detail by Barghouthi et al. (2003).

The $\mathrm{O}^{+}-\mathrm{O}$ collision process was simulated using a combination of the resonant charge exchange and polarization interaction models (Barakat et al., 1983; Barghouthi and Qatanani, 2003). Resonant charge exchange occurs when an electron jumps from a neutral $\mathrm{O}$ atom to a colliding $\mathrm{O}^{+}$ ion. Due to this exchange, the final velocity of the $\mathrm{O}^{+}$ion is the velocity of the neutral $\mathrm{O}$, which generated from a nondrifting Maxwellian distribution with neutral temperature $T_{\mathrm{O}}=1000 \mathrm{~K}$. However, in the polarization interaction the test $\mathrm{O}^{+}$ion interacts with the electric dipole that it induces in the neutral $\mathrm{O}$. In the case of polarization interaction, the scattering process was assumed to be isotropic (i.e. the differential scattering cross section is independent of the scattering angle) and the scattering cross section varies as $g^{-1}$ (Barghouthi et al., 2003; Barghouthi and Qatanani, 2003), where $g=\left|\mathbf{v}_{\mathrm{O}^{+}}-\mathbf{v}_{\mathrm{O}}\right|$ is the relative speed between colliding particles $\mathrm{O}^{+}$and $\mathrm{O}$. For polarization interaction collisions, the resultant change in the $\mathrm{O}^{+}$ion's velocity is computed by assuming the collision is elastic and $\mathbf{g}$ is deflected through a random angle uniformly distributed between 0 and $\pi$. The relative velocity $\mathbf{g}$ and the velocity of the center of gravity, $\mathbf{v}_{C G}$, between the colliding particles are computed. As a result of polarization interactions, the magnitude of the relative velocity $\mathbf{g}$ is unchanged but its direction is altered. However, the velocity of the center of gravity remains the same before and after polarization interaction collision. These velocities $\mathbf{v}_{C G}$ and $\mathbf{g}$ are used to calculate the velocity of the test $\mathrm{O}^{+}$ion, $\mathbf{v}_{\mathrm{O}^{+}}^{\prime}$ after polarization interaction (Barghouthi and Qatanani, 2003).

Barghouthi et al. (2003) used this Monte Carlo model to investigate the behavior of $\mathrm{O}^{+}$ions in the auroral ionosphere $(150 \mathrm{~km}-500 \mathrm{~km})$ that are $\mathbf{E} \times \mathbf{B}$ drifting in a background of neutrals $\mathrm{O}$, for different electric field intensities $\left[E=0,50,100,150\right.$ and $\left.200 \mathrm{mVm}^{-1}\right]$. They assumed the distribution function of the neutral $\mathrm{O}$ to be a non-drifting-Maxwellian, with temperature $T_{\mathrm{O}}=1000 \mathrm{~K}$ and the geomagnetic field $B$ was assumed to be 0.5 Gauss. They found, as altitude increases the $\mathrm{O}^{+}{ }_{-} \mathrm{O}^{+}$Coulomb collision becomes significant and hence, the non-Maxwellian features of the $\mathrm{O}^{+}$velocity distribution function are reduced. Also, as electric field increases, the effect of $\mathrm{O}^{+}$$\mathrm{O}^{+}$Coulomb collisions is decreased and the role of $\mathrm{O}^{+}-\mathrm{O}$ collisions becomes important.

In the next section we use the Monte Carlo calculations of the $\mathrm{O}^{+}$velocity distributions (Barghouthi et al., 2003) to compute the incoherent radar spectra at different altitudes and for large value of convection electric field.

\section{Basic Formulation of the Radar Spectrum}

In the following, some important expressions, which can be used to describe the incoherent scatter process at Fregion altitudes, are considered. Using the formulation given by Raman et al. (1981) and Kikuchi et al. (1989), the spectral density function $S(\mathbf{k}, w)$ is given by the expression

$$
S(\mathbf{k}, w)=2\left(H_{e} / \varepsilon\right)^{2} M_{i}+2\left(1-H_{e} / \varepsilon\right)^{2} M_{e}
$$

where

$$
\begin{aligned}
& \varepsilon=1+H_{e}+H_{i} \\
& H_{e}=\alpha^{2}\left[1-y_{e} \exp \left(-y_{e}^{2}\right)\right. \\
& \left.\times\left(i \pi^{1 / 2}+2 \int_{0}^{y_{e}} \exp \left(y^{2}\right) d y\right)\right] \\
& H_{i}=\lim _{\gamma \rightarrow 0} \sum_{i} \frac{4 \pi e^{2}}{m_{i} k^{2}} \int \frac{k d g_{i} / d u}{w-k u-i \gamma} d u \\
& M_{e}=\frac{\pi^{1 / 2}}{k a} \exp \left(-y_{e}^{2}\right) \\
& M_{i}=\frac{\pi}{k} \sum_{i} g_{i}(w / k) \\
& g_{i}(u)=\frac{\int F_{0_{i}} \delta(u-\mathbf{k} \cdot \mathbf{v} / k) d \mathbf{v}}{\int F_{0_{i}} d \mathbf{v}}
\end{aligned}
$$

in these equations

$y_{e}=w / k a, \quad \alpha^{2}=1 /\left(k^{2} \lambda_{d}^{2}\right), \quad$ and $\quad \mathbf{k}=\mathbf{k}_{s}-\mathbf{k}_{i}$

where $\mathbf{k}_{s}$ and $\mathbf{k}_{i}$ are the scattered and incident wave vectors respectively, $H_{i}$ and $M_{i}$ are related to $\mathrm{O}^{+}$ions, $H_{e}$ and $M_{e}$ are related to the electrons; $\lambda_{d}$ is the Debye length, $\alpha=\left(2 k_{B} T_{e} / m_{e}\right)^{1 / 2}$ is the electron thermal speed, $w$ is the Doppler shift of the incident frequency, $F_{O_{i}}$ is the 3-D O ${ }^{+}$ velocity distribution function, and $g_{i}$ is the one-dimensional $\mathrm{O}^{+}$velocity distribution function, along the $\mathbf{k}$ (line-of-sight) direction.

The shape of the 1- $\mathrm{D} \mathrm{O}^{+}$distribution function in a background of neutrals $\mathrm{O}$, in the high latitude $\mathrm{F}$-region and in the presence of high electric fields has been extensively studied by Kikuchi et al. (1989), Barakat and Hubert (1990), Winkler et al. (1992), Hubert and Leblanc (1997) and Gaimard et al. (1998). These papers were interested in modeling the $1-\mathrm{D} \mathrm{O}^{+}$distribution function for the derivation of the plasma parameters from incoherent radar spectra. These studies neglected the effect of $\mathrm{O}^{+}-\mathrm{O}^{+}$Coulomb collisions. In this paper we precisely determine the shape of 1-D $\mathrm{O}^{+}$ velocity distribution function, $g_{i}$, along the radar line-ofsight from the 3-dimensional Monte Carlo simulation which takes into account the effects of $\mathrm{O}^{+}-\mathrm{O}$ collisions as well as $\mathrm{O}^{+} \mathrm{O}^{+}$Coulomb collisions in the presence of large convection electric field. A spline fit is used to filter out the noise due to the Monte Carlo method. The smoothed 1-D O $\mathrm{O}^{+}$ velocity distribution function, $g_{i}$, is used directly in equations (4-10) to find the radar spectrum for different values of electron temperature $T_{e}$.

\section{Incoherent Radar Spectra}

The 3-D $\mathrm{O}^{+}$velocity distribution function was computed directly from the Monte Carlo simulation. The $1-\mathrm{D} \mathrm{O}^{+}$ve- 
locity distribution along a given direction is directly computed via a 1-D grid with equally-wide bins along that direction. In comparison to the 3-D grid, the 1-D grid can have more bins per thermal speed and larger number of $\mathrm{O}^{+}$ ions per bin. This result is a significant improvement in both the resolution and accuracy of the produced $1-\mathrm{D} \mathrm{O}^{+}$velocity distribution. The associated error is estimated for each value computed (i.e. value of the velocity distribution function at a given velocity $v$ ). This is achieved by dividing the sequence of the random numbers into several subsequences $\left\{s_{1}, \ldots, s_{n}\right\}$, such that each sequence simulated the same number of collisions. For a given variable $y$ we compute the mean value $\left\{y_{i}, \ldots, y_{n}\right\}$ associated with the corresponding subsequences. Then the overall mean $\bar{y}$ and standard deviation $\sigma_{y}$ are found from

$$
\begin{aligned}
\bar{y} & =\frac{1}{n} \sum_{i=1}^{n} y_{i} \\
\sigma_{y} & =\frac{1}{n}\left[\sum_{i=1}^{n}\left(y_{i}-\bar{y}\right)^{2}\right]^{1 / 2}
\end{aligned}
$$

The error estimation should not depend critically on the number of subsequences, $n$. As a check, we compared the results with $n=100$ and $n=1000$, and we found the error estimates for the two cases to be similar. The accuracy of this algorithm was tested by running the Monte Carlo code for some special cases where the exact results are known.

Figure 1 shows a comparison between 1-D $\mathrm{O}^{+}$velocity distribution functions for different typical values of $\mathrm{O}^{+}$-to$\mathrm{O}$ density ratio $n_{\mathrm{O}^{+}} / n_{\mathrm{O}}=10^{-5}$ (i.e. at $180 \mathrm{~km}$ ) solid line and $n_{\mathrm{O}^{+}} / n_{\mathrm{O}}=10^{-3}$ (i.e. $350 \mathrm{~km}$ ) dotted line, the convection electric field $E$ is $100 \mathrm{mVm}^{-1}$ and the angle between the radar line-of-sight and the geomagnetic field is $60^{\circ}$. As the density ratio $n_{\mathrm{O}^{+}} / n_{\mathrm{O}}$ increases from $10^{-5}$ to $10^{-3}$ (i.e. as altitude increases from $180 \mathrm{~km}$ to $350 \mathrm{~km}$ ) the $1-\mathrm{D} \mathrm{O}^{+}$ velocity distribution function departs from non-Maxwellian at $180 \mathrm{~km}$, due to the effect of $\mathbf{E} \times \mathbf{B}$ which drives $\mathrm{O}^{+}$ ions away from equilibrium, to Maxwellian at $350 \mathrm{~km}$, due

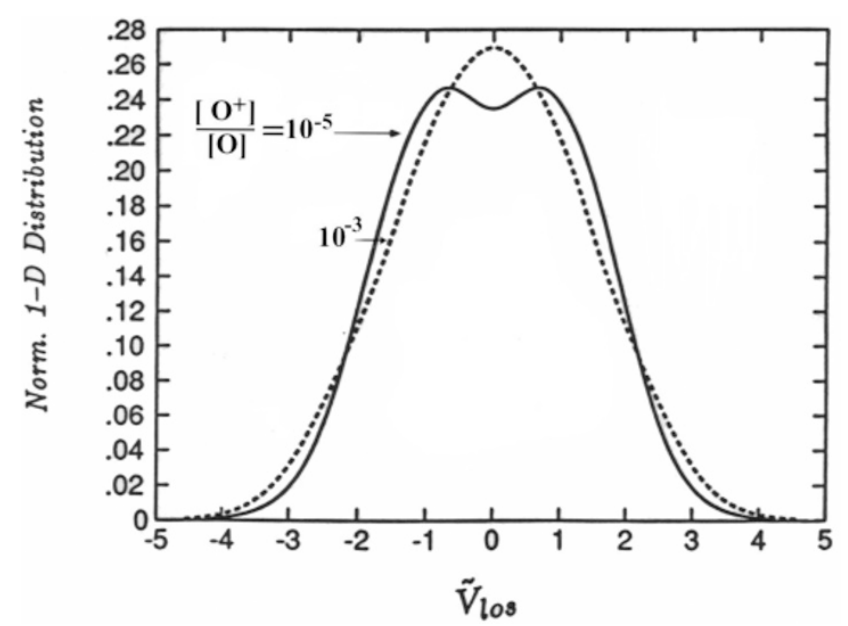

Fig. 1. Comparison between 1-D $\mathrm{O}^{+}$velocity distribution function for different values of ion-to-neutral density ratio $n_{\mathrm{O}^{+}} / n_{\mathrm{O}}\left(10^{-5}\right.$ and $\left.10^{-3}\right)$. The convection electric field, $E$ is equal to $100 \mathrm{mVm}^{-1}$ and the angle between the radar line-of-sight and the geomagnetic field is 60 degrees. to the isotropizing role of $\mathrm{O}^{+}-\mathrm{O}^{+}$Coulomb self-collisions. As altitude increases, the influence of Coulomb collisions $\left(\mathrm{O}^{+}-\mathrm{O}^{+}\right)$becomes more significant. The role of Coulomb collisions is to thermalise (maxwellize) the $\mathrm{O}^{+}$velocity distribution function by transferring thermal energy from the perpendicular direction to the parallel direction (i.e. to isotropize the ion velocity distribution function). This explains the Maxwellian features (one single hump) of the 1$\mathrm{D} \mathrm{O}^{+}$velocity distribution at $350 \mathrm{~km}$, while at low altitude, $(180 \mathrm{~km})$ the $1-\mathrm{D} \mathrm{O}^{+}$velocity distribution has triple humps, which are characteristic of non-Maxwellian velocity distribution (toroidal distribution).

Incoherent scatter spectra for different values of $T_{e}$ (3000, 6000 , and $9000 \mathrm{~K})$, different density ratios $n_{\mathrm{O}^{+}} / n_{\mathrm{O}}\left(10^{-3}\right.$ and $10^{-4}$ ), and for large convection electric field, $E=$ $100 \mathrm{mVm}^{-1}$ are presented in Fig. 2. As the density ratio $n_{\mathrm{O}^{+}} / n_{\mathrm{O}}$ increases from $10^{-5}$ (top panel) to $10^{-3}$ (bottom panel) the features of the spectrum that are characteristics of the non-Maxwellian $\mathrm{O}^{+}$velocity distributions (baby bottle and triple hump) evolve to those that are characteristics of the Maxwellian $\mathrm{O}^{+}$velocity distribution (single hump and double hump). This is due to the effect of $\mathrm{O}^{+}-\mathrm{O}^{+}$Coulomb collisions, which try to thermalise (maxwellize) the $\mathrm{O}^{+}$ velocity distribution function.

The changes in the shape of the Maxwellian spectrum at $\left(n_{\mathrm{O}^{+}} / n_{\mathrm{O}}=10^{-3}\right.$ or altitude $\left.350 \mathrm{~km}\right)$ as a function of

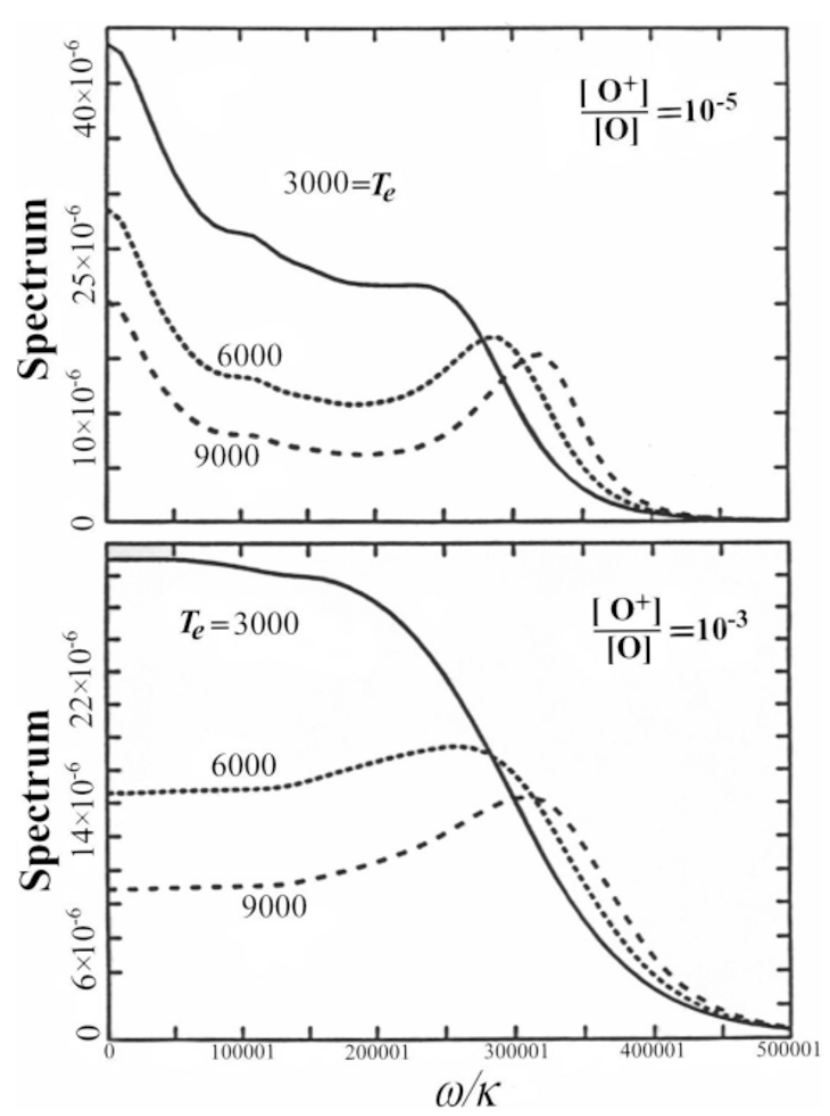

Fig. 2. Incoherent radar spectra for different values of electron temperature $T_{e}(3000,6000$ and $9000 \mathrm{~K})$ and for different ion-to-neutral density ratios $n_{\mathrm{O}^{+}} / n_{\mathrm{O}}\left(10^{-5}\right.$ and $\left.10^{-3}\right)$. The electric field, $E$, is equal to 100 $\mathrm{mVm}^{-1}$ and the angle between the radar line-of-sight and the geomagnetic field is 60 degrees. 
electron temperature $T_{e}$ (Fig. 2 bottom panel) are such that as $T_{e}$ increases, the $\mathrm{O}^{+}$ions acoustic peaks develop and move further apart. The overall spectral intensity decreases slightly. Similar behavior exists for non-Maxwellian spectra (i.e. $n_{\mathrm{O}^{+}} / n_{\mathrm{O}}=10^{-5}$ top panel of Fig. 2).

The changes in the spectral shape as a function of $T_{e}$ for the case $E=100 \mathrm{mVm}^{-1}$ are such that an increase in $T_{e}$ is characterized by an overall decreases in spectral intensity and by the appearance and the shifting apart of the $\mathrm{O}^{+}$ion acoustic peaks. For both cases (top and lower panels of Fig. 2) the width of the spectrum is basically unchanged except for the slight increase caused by an increase in the $\mathrm{O}^{+}$ion acoustic speed. This is consistent with the results of Raman et al. (1981) and Kikuchi et al. (1989). They explained the general behavior of the spectral density in terms of $T_{e} / T_{i}$ and found that as electron temperature, $T_{e}$ increases, the spectral density decreases.

In general, the theoretical predictions given here are supported by several observations. For instance, the toroidal features of the F-region $\mathrm{O}^{+}$velocity distributions were shown to be detectable by a retarding potential analyzer (RPA). The European incoherent scattering radar (EISCAT), with its tristatic mode of operation, was able to measure the $\mathrm{O}^{+}$temperature anisotropy. Perraut et al. (1984) used the EISCAT data to deduce that $\mathrm{O}^{+}$velocity distribution in the auroral F-region was anisotropic. Moreover, clear signatures of non-Maxwellian distributions were observed in the data from the EISCAT radar (Winser et al., 1986; Lockwood et al., 1987). These signatures were similar to those predicted theoretically by Raman et al. (1981).

In spite of the qualitative consistency of the theoretical and observational conclusions, a one-to-one quantitative comparison with experimental data (e.g. McCrea et al., 1993; Ogawa et al., 2000) is not possible for the following reasons. In order to produce an acceptable signalto-noise ratio, the radar output should be integrated over time and space, which limits our ability to uniquely interpret the measurements. Also, the theoretical model is only as reliable as the adopted parameters, such as the differential cross-section. The model presented here also neglects collisions with molecular species (e.g. $\mathrm{NO}^{+}, \mathrm{N}_{2}^{+}, \mathrm{O}_{2}^{+}, \mathrm{N}_{2}$ ) which can be significant in the auroral ionosphere under active conditions, even at F-region heights. Likewise, at high altitudes, the effects of ambipolar, centrifugal and magnetic mirror forces need to be taken into account, and comparison with measured radar spectra should also include the effects of ion upflow. Such factors are hard to account for in this kind of modeling, because their effects are not well enough known. The present study, however, represents the best attempt that can currently be made to reproduce incoherent scatter spectra on the basis of a collision model involving neutral and ionized oxygen.

\section{Conclusions}

We have studied the effect of $\mathrm{O}^{+}-\mathrm{O}^{+}$Coulomb collisions on the spectrum of radar waves incoherently scattered from the disturbed high latitude F-region. The calculations are based on the Monte Carlo simulation of the full 3- $\mathrm{D} \mathrm{O}^{+}$velocity distribution (Barghouthi et al., 2003). The following conclusions can be drawn
1) The 1-D $\mathrm{O}^{+}$velocity distributions computed directly from the Monte Carlo simulation appeared to be Maxwellian at $350 \mathrm{~km}$ and non-Maxwellian at $180 \mathrm{~km}$ for convection electric field $E=100 \mathrm{mVm}^{-1}$ and for typical ion-to-neutral density ratios. The role of $\mathrm{O}^{+}$$\mathrm{O}^{+}$Coulomb collisions becomes significant in thermalising (maxwellizing) the $\mathrm{O}^{+}$velocity distribution function at high altitudes $(\sim 350 \mathrm{~km})$.

2) As the ion-to-neutral density ratio $n_{\mathrm{O}^{+}} / n_{\mathrm{O}}$ increases (i.e. the altitude increases), the features of the spectrum change from baby bottle and triple hump to single and double humps (i.e. the characteristics of the nonMaxwellian distribution evolve to the characteristics of Maxwellian distribution) and this is due to the effect of $\mathrm{O}^{+}-\mathrm{O}^{+}$Coulomb self-collisions.

3) The behavior of Maxwellian spectra and nonMaxwellian spectrum are similar in the case of increasing electron temperature $T_{e}$, in other words the behavior of the spectrum as a function of electrons temperature is slightly changed with altitude.

Acknowledgments. The author would like to thank the referees for their critical reading of the manuscript and constructive suggestions.

\section{References}

Barakat, A. R. and D. Hubert, Comparison of Monte Carlo simulation and polynomial expansions of auroral non-Maxwellian distributions, 2, the 1-D representation, Ann. Geophysicae, 8, 697-704, 1990.

Barakat, A. R., D. Hubert, and J. P. St.-Maurice, Generating a synthetic incoherent scattering radar spectrum using a Monte Carlo simulation, EoS, 71, 1503, 1990.

Barakat, A. R., R. W. Schunk, and J. P. St.-Maurice, Monte Carlo calculations of velocity distributions in the auroral ionosphere, J. Geophys. Res., 88, 3237-3241, 1983.

Barghouthi, I. A. and N. A. Qatanani, Monte Carlo simulation of Maxwell molecule interactions in space plasma, Indian J. Phys., 77B(2), 241245, 2003.

Barghouthi, I. A., A. R. Barakat, and R. W. Schunk, Effect of ion self collisions on the non-Maxwellian ion velocity distributions in the highlatitude F-region, $\operatorname{EoS}, \mathbf{7 2}, 365,1991$.

Barghouthi, I. A., A. R. Barakat, and R. W. Schunk, A Monte Carlo simulation of the effect of ion self-collisions on the ion velocity distribution function in the high-latitude F-region, Ann. Geophysicae, 12, 10761084, 1994.

Barghouthi, I. A., E. I. Elias, M. A. Abu Samra, N. A. Qatanani, and M. S. Issa, Monte Carlo simulation of $\mathrm{O}^{+}$behavior in the auroral ionosphere, J. Phys. Soc. Jpn., 72(11), 2003.

Cole, K. D., Atmospheric excitation and ionization by ions in strong auroral and man-made electric fields, J. Atmos. Terr. Phys., 33, 1241-1249, 1971.

Gaimard, P., C. Lathuillere, and D. Hubert, Non-Maxwellian studies in the auroral F-region: a new analysis of incoherent scatter spectra, J. Atmos. Terr. Phys., 58, 415-433, 1996.

Gaimard, P., J. P. St.-Maurice, C. Lathuillere, and D. Hubert, On the improvement of analytical calculations of collisional auroral ion velocity distributions using recent Monte Carlo results, J. Geophys. Res., 103, 4079-4095, 1998.

Hubert, D., Auroral ion velocity distribution function: Generalized polynomial solution of Boltzmann equation, Planet. Space Sci., 31, 119-127, 1983.

Hubert, D., Non-Maxwellian velocity distribution functions and incoherent scattering of radar waves in the auroral ionosphere, J. Atmos. Terr. Phys., 46, 601-611, 1984.

Hubert, D. and A. R. Barakat, Comparison of Monte Carlo simulation and polynomial expansion of auroral Non-Maxwellian distributions, Part 1: the 3-D representation, Ann. Geophysicae, 8, 687-696, 1990. 
Hubert, D. and F. Leblanc, The auroral $\mathrm{O}^{+}$non-Maxwellian velocity distribution function revisited, Ann. Geophysicae, 15, 249-254, 1997.

Hubert, D., N. Bonnard, C. Lathuillere, and W. Kofman, A new scenario for the measurement of the auroral plasma parameters in the nonMaxwellian state, Geophys. Res. Lett., 20, 2691-2694, 1993.

Kikuchi, K., J. P. St.-Maurice, and A. R. Barakat, Monte Carlo computations of F-region incoherent radar spectra at high latitudes and the use of a simple method for non-Maxwellian spectral calculations, Ann. Geophysicae, 7, 183-194, 1989.

Kinzelin, E. and D. Hubert, Ion velocity distribution function in the upper auroral F-region. 1. Phenomenological approach, J. Geophys. Res., 97, 4061-4072, 1992.

Lockwood, M., B. J. I. Bromage, R. B. Horne, J. P. St.-Maurice, D. Willis, and S. W. H. Cowley, Non-Maxwellian ion velocity distributions observed using EISCAT, Geophys. Res. Lett., 14, 111-114, 1987.

McCrea, I. W., M. Lester, T. R. Robinson, J. P. St.-Maurice, N. M. Wade, and T. B. Jones, Derivation of the ion temperature partition coefficients from the study of ion frictional heating events, J. Geophys. Res., 98, 15,701-15,715, 1993.

Ogawa, Y., R. Fujii, S. C. Buchert, S. Nozawa, S. Watanabe, and A. P. van Eyken, Simultaneous EISCAT Svalbard and VHF radar observations of ion upflows at different aspect angles, Geophys. Res. Lett., 27(1), 81-84, 2000.

Perraut, S., A. Brekke, M. Baron, and D. Hubert, EISCAT measurements of ion temperatures which indicate non-isotropic ion velocity distributions, J. Atoms. Terr. Phys., 46, 531-543, 1984.

Raman, R. S. V., J. P. St-Maurice, and R. S. B. Ong, Incoherent scattering of radar waves in the auroral ionosphere, J. Geophys. Res., 86, 4751-
4762, 1981.

Salah, J. E., Interim standard for the ion-neutral atomic oxygen collision frequency, Geophys. Res. Lett., 20, 1543-1546, 1993.

St.-Maurice, J. P. and R. W. Schunk, Auroral ion velocity distributions using a relaxation model, Planet. Space Sci., 21, 1115-1130, 1973.

St.-Maurice, J. P. and R. W. Schunk, Use of generalized orthogonal polynomial solutions of Boltzmann equation in certain aeronomy problems: auroral ion velocity distributions, J. Geophys. Res., 81, 2145-2154, 1976.

St.-Maurice, J. P. and R. W. Schunk, Ion velocity distributions in the highlatitude ionosphere, Rev. Geophys. Space Phys., 17, 99-133, 1979.

Takisuka, T. and H. Abe, A binary collision model for plasma simulation with a particle code, J. Comp. Phys., 25, 205-219, 1977.

Tereshchenko, V. D., E. D. Tereshchenko, and H. Kohl, The incoherent scattering of radio waves in a non-Maxwellian plasma: The effect of Coulomb collisions, J. Geophys. Res., 96, 17,591-17,598, 1991.

Winkler, E., J. P. St.-Maurice, and A. R. Barakat, Results from improved Monte Carlo calculations of auroral ion velocity distributions, J. Geophys. Res., 97, 8399-8423, 1992.

Winser, K. J., G. O. L. Jones, and P. J. S. Williams, A quantitative study of the high-latitude ionospheric trough using EISCAT's common programs, J. Atoms. Terr. Phys., 48, 893-904, 1986.

Winser, K. J., M. Lockwood, and G. O. L. Jones, Non-thermal plasma observations using EISCAT: Aspect angle dependence, Geophys. Res. Lett., 14, 957-960, 1987.

I. A. Barghouthi (e-mail: barghouthi2@yahoo.com) 